\title{
Impact of Exposure to Dim Artificial Light at Night during Sleep on Cognitive Function
}

\author{
Younjung Lee', Chul-Hyun Cho ${ }^{2,3,4}$, and Heon-Jeong Lee ${ }^{4,5}$ \\ 'Department of Psychiatry, Gyeongsang National University School of Medicine and Gyeongsang National University Hospital, Jinju, Korea \\ 2Department of Psychiatry, College of Medicine, Chungnam National University, Daejeon, Korea \\ ${ }^{3}$ Department of Psychiatry, Chungnam National University Sejong Hospital, Sejong, Korea \\ ${ }^{4}$ Chronobiology Institute, Korea University, Seoul, Korea \\ ${ }^{5}$ Department of Psychiatry, Korea University College of Medicine, Seoul, Korea
}

Objective: Artificial light at night can impede sleep quality and can cause sleep disturbances. This study aimed to determine if dim artificial light at night (dALAN) affects cognitive function and motor performance. Methods: A total of 30 healthy young male volunteers aged 21 to 29 years were enrolled in the study. They were randomly divided into two groups depending on light intensity (Group A: 5 lux and Group B: 10 lux). After a quality control process, 23 healthy subjects were included in the study (Group A: 11 subjects, Group B: 12 subjects). Data were gathered from each participant after each night with no light (Night 1) followed by the next night (Night 2) with two different dim light conditions ( 5 or 10 lux) using a computerized neurocognitive function test (Vienna test). Results: In the cognitrone test, there was a significant difference in the mean time of correct Yes-response $(F=9.08, p=0.007)$ and the mean time of correct No-response $(F=7.12, p=0.014)$ in both Group A (5 lux) and Group B (10 lux) before and after exposure to dALAN. In the vigilance and motor performance series tests, there were no significant differences before and after exposure to dALAN in both groups, and no differences were observed between the groups. Conclusion: We found that dALAN exposure did not have a significant effect on cognition, vigilance, or motor performance; rather, the speed was faster in the cognition test after dALAN exposure.

Key Words: Dim artificial light at night; Vienna test; Sleep; Cognitive function

Received: November 17, 2020 Revised: December 1, 2020 Accepted: December 3, 2020

Corresponding author: Chul-Hyun Cho, MD, PhD, Department of Psychiatry, College of Medicine, Chungnam National University, 82 Munhwa-ro, Jung-gu, Daejeon 35015, Korea.

Tel: 82-44-995-4775, Fax: 82-0504-056-2203, E-mail: david0203@gmail.com

(a) This is an Open Access article distributed under the terms of the Creative Commons Attribution Non-Commercial License (https://creativecommons.org/licenses/bync/4.0) which permits unrestricted non-commercial use, distribution, and reproduction in any medium, provided the original work is properly cited.

\section{INTRODUCTION}

Sleep is a complex interaction with various brain structures, neurotransmitter systems, and regulatory hormones [1]. It is a reversible and repetitive action that includes roles such as recovery and growth, learning and memory reinforcement, and restorative processes [2]. Therefore, sleep is an essential element of human life that maintains a circadian rhythm of day and night [3]. Any problems with the amount and quality of sleep can affect the human circadian rhythm, mood, activity, and productivity $[4,5]$. In addition, sleep deprivation and sleep deterioration affect not only human daily life, but also the incidence and exacerbation of various psychiatric disorders such as mood disorders, anxiety disorders, schizophrenia, and neurodegenerative disorders such as dementia [1]. In particular, sleep is known to be closely related to cognitive function and memory. Yoo et al. [6] reported that sleep is an essential element in the process of learning and memory, and it is important to sleep before learning to consolidate memory. Sleep deprivation in animal studies interferes with the acquisition of learning and causes problems with hippocampus-related performance $[7,8]$.

According to various studies, lack of sleep affects the daily life of humans and causes a decrease in various performance functions. Pilcher and Huffcutt [9] found that sleep deprivation affects motor performance, cognitive function, and mood. Among them, mood change is the most severe and decreases in the order of cognitive function and motor performance. However, it was explained that accurate evaluation is difficult because mood chang- 
es are often measured by subjective reports. In addition, sleep deprivation could have a negative impact not only on a cognitive function, but also on responding to unexpected events such as revising or innovating plans; it also damages effective communication. According to Curcio et al. [2], the quality and quantity of sleep are closely related to students' learning ability and academic performance. They reported that learning performance could deteriorate if sleep was deprived. They also suggested that sleep deprivation is related to prefrontal cortex function. As such, sleep is closely related to the daily life of people, and sleep loss is not only related to diseases, but also affects daily life such as memory, mood, and stress [10]. People are greatly affected by the sleep cycle due to artificial light at night (ALAN). People enjoy convenience due to artificial light at night; however, as artificial light became more common, people were exposed to unwanted ALAN. ALAN impedes sleep quality, and sleep disturbances caused by ALAN, when absolute darkness is required, have recently been considered to be light pollution [11]. Even when the lights in the bedroom are switched off, exposure to dim artificial light at night (dALAN), which is light leaking from outside through a window, can be overlooked by people. Fonken et al. [12] reported that exposure to dALAN in animal experiments affects cognitive function and mood. There are reports that dALAN affects the structure of sleep and increases the frequency of wakefulness and the amount of REM sleep [13]. As such, studies on the effect of dALAN on cognitive function have been mainly conducted through animal experiments, and there are not many studies on humans. We hypothesized that dALAN affects cognitive function and motor performance.

\section{METHODS}

\section{Participants}

A total of 30 adult men ranging in age from 19 to 29 years (mean age $\pm S D: 22.26 \pm 2.65)$ were recruited for this study through Internet bulletin boards and staff referrals. We chose only young healthy male participants to eliminate age, sex, and health-related confounders. All participants completed a questionnaire regarding their sleeping conditions, physical health, and psychiatric health prior to participation. A total of 30 participants were selected and randomly classified into two groups according to the light intensity they were exposed to at nighttime (Group A: 5 lux, Group B: 10 lux). Participants were asked to wear an actiwatch (Actiwatch-L, Mini Mitter Co. Inc., Bend, OR, USA) on their wrists from 1 week before the experiment, and their circadian rhythm was analyzed. Participants who did not sleep at night or had excessive sleep during the day were excluded. In addition, participants with sleep efficiency $<90 \%$ or apnea-hypopnea index $>5$ were excluded from the nocturnal polysomnography (NPSG) test conducted on the first day of the experiment. Finally, 23 healthy participants were included in the study: 11 in Group A and 12 in Group B. Each participant provided informed written consent, which was approved by the Institutional Review Board (IRB) of the Korea University Anam Hospital (IRB NO. ED12261), before participating in the study.

\section{Protocol}

From 1 week before the study, all subjects were allowed to sleep only at night without sleeping during the day to maintain a regular circadian rhythm, and this was confirmed by actigraph records. All subjects were banned from consuming medicines, coffee, alcohol, and other substances that may affect sleep. NPSG was performed for three consecutive nights. On the first day of the study (Night 0), subjects underwent NPSG with no ALAN to reduce its effect on that timepoint. On the second day (Night 1), subjects slept in a sleep test room without ALAN, and the NPSG was performed. On the third day (Night 2), subjects underwent an NPSG session with dim light of 5 lux or 10 lux according to the group classified. On the third day, the dim light box (daylight "cool white" color) was placed above the wall opposite the subject's head. For each NPSG session of the third night, the luminous intensity was checked by an illuminometer (ANA-F11, Tokyo Photo Electric Co., Ltd., Tokyo, Japan) at the level of the participant's eyes while supine. Light intensity was set to 5 lux (Group A) or 10 lux (Group B) based on random assignment; participants were not informed whether the dALAN was 5 lux or 10 lux. dALAN was maintained the whole night for the third day's (Night 2) NPSG session. During the entire study period, participants were instructed to sleep in the supine position as much as possible and not to cover their face with a blanket.

\section{Measurements}

Cognitive function tests were performed the day after sleep on the second day (Night 1) and the third day (Night 2). In this study, the Vienna test (Dr G. Schufried, Moedling, Austria), a representative method for cognitive function evaluation, was performed as a kind of computerized neurocognitive function test to confirm the change in cognitive function [14].

\section{Cognitrone test}

This is a complex task that measures continuous attention, cognitive flexibility, visual immediate memory, and information processing speed. The method compares four figures presented at the top of the screen and one figure at the bottom to determine whether they are the same thing.

\section{Vigilance test}

This test examined whether the subject's alertness level is maintained even in the state by providing only low-frequency simple stimulation for a long time. A large circle made of small circles appeared on the screen. While the subject looked at the screen, a white circle moved one space at a time; when it moved two spaces, the subject pressed the black button.

\section{Motor performance test series}

This is a test to evaluate the fine motor control ability of the up- 
per limb. It uses electronic test equipment to objectively assess minute motor skills to assess the maximum number of minute motor activity factors, which should be relevant to practical work.

Steadiness (practice phase)

The subject held a black pen and was in the state of putting it halfway into the largest hole ( $8.5 \mathrm{~mm}$ in diameter) among the life preservers in the top row. The pen was not allowed to touch the bottom or wall of the hole at this time. The test started when the inspector pressed the enter key.

Line tracking (practice phase)

The subject held a black pen, lightly hit the bottom of the circle at the right end (start point) of the maze in the center of the instrument, and then moved it to the left along the maze. At this time, the pen tip was prevented from touching the floor or wall of the maze as much as possible. The subject took the pen to the circle at the far left and hit the floor lightly again. The test began when the subject touched the starting point.

\section{Aiming}

The subject grabbed a black pen and quickly hit the pen tip lightly from the right to the left of the upper row of small silver circles set in two rows. This test was also initiated by the subject.

\section{Inserting long pins}

The subject placed a long pin $10 \mathrm{~cm}$ away from the instrument plate and inserted the pins one by one from the bottom hole to the top hole. This test began when the subject inserted a pin into the bottom hole and ended when the subject inserted it into the last hole. The subject performed the test as quickly as possible.

\section{Tapping}

The subject held a black pen and hit the square plate at the bottom of the instrument plate lightly and quickly for $32 \mathrm{sec}$. At this time, the subject held the pen underneath and maintained it as vertically as possible.

\section{Steadiness}

This time, the subject was asked to insert half a black pen into the smallest hole ( $4.8 \mathrm{~mm}$ in diameter) of the upper row and hold it exactly vertically. The subject pressed the enter key to start the test.

\section{Line tracking}

Subjects performed the same as in the practice phase.

\section{Data analysis}

We used Student's t-test or Mann-Whitney U test to compare demographic characteristics and cognitive function evaluation scale variables. Demographic characteristics included age, educational level, weight, and height. To determine the effect of dALAN differences between groups and night-group interactions, we performed repeated measures analysis of variance on the $\mathrm{Vi}$ enna test results of Night 1 and 2, respectively. All statistical analyses were performed using IBM SPSS software (Version 24.0, IBM Corp., Armonk, NY, USA). The significance level was set at $\mathrm{p}<0.05$ (two-tailed).

\section{RESULTS}

In this study, the effect of dim light exposure during night sleep on cognitive function on the next day was evaluated through analysis of light intensity before and after light exposure. There was no significant difference in demographic characteristics between the two groups. Demographic characteristics include age, education level, weight, height. Basic information including demographic characteristics was presented through the previous study conducted with the same research method [15].

\section{Cognitrone test}

There was a significant difference in mean time correct Yes-response in both Group A (5 lux) and Group B (10 lux) (F=9.08, $\mathrm{p}=0.007)$ before and after exposure to dALAN. In both groups, the mean time correct Yes-response decreased on Night 2, that is, the test performed after dALAN, compared to the test result performed on Night 1 . There was a difference over time, but no difference between groups was found $(\mathrm{F}=1.45, \mathrm{p}=0.241)$. In addition, the mean time of correct No-response also showed a significant difference in both groups before and after exposure to light, and mean time decreased on Night $2(\mathrm{~F}=7.12, \mathrm{p}=0.014)$. However, the number of correct Yes-response, number of correct No-response, and mean time of correct No-response did not differ significantly between groups before and after exposure to dALAN (Table 1).

\section{Vigilance test}

In the vigilance test, the number of correct and mean values of reaction time were measured. There were no significant differences before and after exposure to dALAN in both groups, and no differences were observed between groups (Table 1).

\section{Motor performance test series}

In the motor performance test, there was no difference between groups in all areas and the time before and after exposure to dALAN (Table 1).

\section{DISCUSSION}

Acute and partial sleep deprivation and sleep quality negatively affect cognitive function, vigilance, motor control ability, and mood [16]. Therefore, maintenance of sleep quality and circadian rhythm is essential for humans, and sleep quality deterioration and fluctuations in circadian rhythm can have a negative effect on humans. A study has shown that the leakage of light at night inhibits the secretion of melatonin and has a negative effect on the 
Table 1. Results of a repeated measures analysis of variance on cognitive tasks between days and groups

\begin{tabular}{|c|c|c|c|c|c|c|}
\hline \multirow{2}{*}{ Variables } & \multirow{2}{*}{ Nights } & Group A $(n=11)$ & Group B $(n=12)$ & \multirow{2}{*}{ Source } & \multirow{2}{*}{$\mathrm{F}$} & \multirow{2}{*}{$\mathrm{p}$} \\
\hline & & Mean \pm SD & Mean \pm SD & & & \\
\hline \multicolumn{7}{|l|}{ Cognitrone test } \\
\hline \multirow[t]{2}{*}{ n.CYR } & Night 1 & $76.09 \pm 4.16$ & $75.25 \pm 4.16$ & Nights & 0.24 & 0.630 \\
\hline & Night 2 & $75.18 \pm 5.23$ & $75.58 \pm 3.58$ & Night $\times$ Group & 1.11 & 0.304 \\
\hline \multirow[t]{2}{*}{ n.CNR } & Night 1 & $111.27 \pm 12.97$ & $117.17 \pm 2.92$ & Nights & 0.16 & 0.696 \\
\hline & Night 2 & $111.09 \pm 14.12$ & $117.00 \pm 3.59$ & Night $\times$ Group & $<0.01$ & 0.986 \\
\hline \multirow[t]{2}{*}{ mt.CYR } & Night 1 & $1.34 \pm 0.15$ & $1.35 \pm 0.20$ & Nights & 9.08 & $0.007^{*}$ \\
\hline & Night 2 & $1.30 \pm 0.17$ & $1.26 \pm 0.20$ & Night $\times$ Group & 1.45 & 0.241 \\
\hline \multirow[t]{2}{*}{ mt.CNR } & Night 1 & $1.53 \pm 0.20$ & $1.45 \pm 0.25$ & Nights & 7.12 & $0.014^{*}$ \\
\hline & Night 2 & $1.47 \pm 0.19$ & $1.37 \pm 0.24$ & Night $\times$ Group & 0.37 & 0.549 \\
\hline \multicolumn{7}{|l|}{ Vigilance test } \\
\hline \multirow[t]{2}{*}{ n.Cor } & Night 1 & $97.55 \pm 3.24$ & $93.67 \pm 4.70$ & Nights & 0.28 & 0.604 \\
\hline & Night 2 & $96.73 \pm 3.82$ & $93.75 \pm 5.31$ & Night $\times$ Group & 0.42 & 0.525 \\
\hline \multirow[t]{2}{*}{ mv.RxnTime } & Night 1 & $0.51 \pm 0.08$ & $0.53 \pm 0.08$ & Nights & 0.06 & 0.814 \\
\hline & Night 2 & $0.50 \pm 0.08$ & $0.55 \pm 0.07$ & Night $\times$ Group & 1.66 & 0.211 \\
\hline \multicolumn{7}{|l|}{ Motor performance test } \\
\hline \multirow[t]{2}{*}{ Steadiness E } & Night 1 & $0.36 \pm 0.67$ & $2.17 \pm 3.69$ & Nights & 1.77 & 0.197 \\
\hline & Night 2 & $0.36 \pm 0.67$ & $0.67 \pm 0.78$ & Night $\times$ Group & 1.77 & 0.197 \\
\hline \multirow[t]{2}{*}{ Steadiness EL } & Night 1 & $0.03 \pm 0.06$ & $0.17 \pm 0.28$ & Nights & 2.31 & 0.144 \\
\hline & Night 2 & $0.04 \pm 0.08$ & $0.02 \pm 0.04$ & Night $\times$ Group & 3.12 & 0.092 \\
\hline \multirow[t]{2}{*}{ Line tracking $\mathrm{E}$} & Night 1 & $19.91 \pm 3.89$ & $20.83 \pm 6.37$ & Nights & $<0.01$ & 0.973 \\
\hline & Night 2 & $18.36 \pm 5.33$ & $22.50 \pm 12.75$ & Night $\times$ Group & 0.85 & 0.366 \\
\hline \multirow[t]{2}{*}{ Line tracking EL } & Night 1 & $1.18 \pm 0.55$ & $1.25 \pm 0.56$ & Nights & 0.86 & 0.364 \\
\hline & Night 2 & $1.12 \pm 0.49$ & $1.57 \pm 1.02$ & Night $\times$ Group & 1.68 & 0.209 \\
\hline \multirow[t]{2}{*}{ Line tracking $\mathrm{T}$} & Night 1 & $19.69 \pm 9.45$ & $17.94 \pm 4.46$ & Nights & 0.96 & 0.338 \\
\hline & Night 2 & $19.63 \pm 9.15$ & $19.87 \pm 7.07$ & Night $\times$ Group & 1.11 & 0.304 \\
\hline \multirow[t]{2}{*}{ Aiming E } & Night 1 & $0.55 \pm 0.69$ & $0.42 \pm 0.52$ & Nights & 0.37 & 0.548 \\
\hline & Night 2 & $0.45 \pm 0.82$ & $0.33 \pm 0.50$ & Night $\times$ Group & $<0.01$ & 0.979 \\
\hline \multirow[t]{2}{*}{ Aiming $\mathrm{H}$} & Night 1 & $19.82 \pm 0.87$ & $19.83 \pm 0.40$ & Nights & 0.05 & 0.830 \\
\hline & Night 2 & $19.73 \pm 0.65$ & $20.00 \pm 0.00$ & Night $\times$ Group & 0.55 & 0.467 \\
\hline \multirow[t]{2}{*}{ Aiming EL } & Night 1 & $0.02 \pm 0.03$ & $0.018 \pm 0.02$ & Nights & $<0.01$ & 0.929 \\
\hline & Night 2 & $0.03 \pm 0.05$ & $0.02 \pm 0.03$ & Night $\times$ Group & 0.04 & 0.849 \\
\hline \multirow[t]{2}{*}{ Aiming $\mathrm{T}$} & Night 1 & $7.52 \pm 1.18$ & $6.87 \pm 1.13$ & Nights & 0.33 & 0.568 \\
\hline & Night 2 & $7.30 \pm 1.50$ & $6.90 \pm 1.44$ & Night $\times$ Group & 0.54 & 0.471 \\
\hline \multirow[t]{2}{*}{ Tapping H } & Night 1 & $201.64 \pm 24.48$ & $211.67 \pm 41.51$ & Nights & 3.63 & 0.071 \\
\hline & Night 2 & $196.45 \pm 23.54$ & $202.67 \pm 27.46$ & Night $\times$ Group & 0.26 & 0.613 \\
\hline \multirow[t]{2}{*}{ Inserting long pins $\mathrm{T}$} & Night 1 & $33.67 \pm 2.80$ & $34.40 \pm 3.31$ & Nights & 0.37 & 0.550 \\
\hline & Night 2 & $33.61 \pm 4.09$ & $35.07 \pm 3.80$ & Night $\times$ Group & 0.52 & 0.480 \\
\hline
\end{tabular}

${ }^{*} \mathrm{p}<0.05$. n.CYR: number of correct Yes-response, n.CNR: number of correct No-response, mt.CYR: mean time of correct Yes-response (sec), mt.CNR: mean time of correct No-response (sec), n.Cor: number of correct, mv.RxnTime: mean value of reaction time (sec), E: number of errors, EL: length of error (sec), H: number of hits, T: total time (sec), SD: standard deviation

decrease in thermoregulation, glucose homeostasis, and blood pressure control, as well as human circadian pacemakers, when chronic exposure is present [17].

According to Cho et al. [15], subjective cognitive function impairment or decreased concentration was reported in Group B compared to Group A in an experiment conducted by subjects as in this study. Based on the results of several studies, we hypothesized that exposure to dALAN would have a negative effect on sleep and intensify fatigue, which will affect cognitive function and motor performance. But we found that dALAN exposure did not have a significant effect on cognition, vigilance, and motor performance; rather, the speed was faster in the cognition test after dALAN exposure. Kang et al. [18] reported that the activity of the right and left inferior frontal gyrus of the brain decreases during working memory performance when exposed to 10 lux of light during nighttime sleep. These results support the fact that weak light exposure at night reduces the brain function responsible for memory. However, some studies have shown that one night of sleep deprivation affects psychological wellbeing and executive functions, but does not significantly affect simple reaction time 
and speed of psychomotor or motor performance [19]. This result is consistent with the current study, which also showed that there is no significant effect on cognitive function and motor performance task due to one day of nighttime light exposure. However, in this study, we found that the performance speed of the cognitive function evaluation test was further improved on Night 2 compared to Night 1 . Since this test is relatively simple, it can be considered a result of the learning effect by repetition [20]. The Vienna test is a computer-based test where the test stimulus was kept constant, the response was accurately recorded, the cost and time were reduced, and the subjective factors of the tester were not involved [21]. However, this computerized neurocognitive function test measures the ability to perform relatively simple tasks. According to previous studies, the cognitive function measured by this test easily deteriorated in the task of alertness to a simple stimulus. However, it is reported that more complex and higher-level cognitive function tests are less affected [9]. Regarding the cognitrone test, which measures visual perception analysis, attention, cognitive flexibility, and stress endurance, there have been studies that reported results of improved performance after sleep deprivation $[22,23]$. This result can be attributed to the effect of practice obtained as a result of repeated performance. Another possible explanation is that the subject's compensatory efforts may have been intervened during the process of cognitive function higher than the simple response. It is also possible that the subject controlled the level of arousal to perform something when feeling sleepy [20]. This possibility is thought to explain the results of this study.

This study has several limitations. First, the number of samples was rather small. Owing to the nature of polysomnography, it is difficult to perform a number of polysomnography studies. In the future, if possible, better results can be obtained by conducting research with a larger number of subjects. The second limitation is that only two methods were selected: the cognitrone test and the vigilance test, which measures simple attention. In the future, it will be helpful to add tests for cognitive function areas such as high-level analysis, reaction tests, and short-term memory tests. Finally, the experimental design of this study was not able to control for practice effects despite the use of familiar procedures. In the future, an in-depth study on sleep quality deterioration caused by dALAN is necessary by supplementing the limitations of this study.

\section{Acknowledgments}

This work was supported by the National Research Foundation of Korea (NRF) grant funded by the Government of Korea (MSIT) (NRF-2020R1C1C1007463), and the Brain Research Program through the National Research Foundation of Korea (NRF) funded by the Ministry of Science, ICT \& Future Planning (NRF2016M3C7A1914448).

\section{Conflicts of Interest}

The authors have no potential conflicts of interest to disclose.

\section{Author Contributions}

Conceptualization: Chul-Hyun Cho, Heon-Jeong Lee. Data curation: Chul-Hyun Cho, Heon-Jeong Lee. Formal analysis: Younjung Lee, Chul-Hyun Cho, Heon-Jeong Lee. Funding acquisition: Chul-Hyun Cho, Heon-Jeong Lee. Investigation: Younjung Lee, Chul-Hyun Cho, Heon-Jeong Lee. Methodology: Chul-Hyun Cho, Heon-Jeong Lee. Project administration: Chul-Hyun Cho, Heon-Jeong Lee. Resources: Chul-Hyun Cho, Heon-Jeong Lee. Software: Chul-Hyun Cho, Heon-Jeong Lee. Supervision: ChulHyun Cho, Heon-Jeong Lee. Validation: Younjung Lee, ChulHyun Cho. Visualization: Younjung Lee, Chul-Hyun Cho. Writing-original draft: Younjung Lee, Chul-Hyun Cho. Writingreview \& editing: Younjung Lee, Chul-Hyun Cho, Heon-Jeong Lee.

\section{ORCID iDs}

Younjung Lee (1)

https://orcid.org/0000-0003-4208-7039

Chul-Hyun Cho (1)

https://orcid.org/0000-0002-1663-9680

Heon-Jeong Lee (1)

https://orcid.org/0000-0002-9560-2383

\section{REFERENCES}

1. Wulff K, Gatti S, Wettstein JG, Foster RG. Sleep and circadian rhythm disruption in psychiatric and neurodegenerative disease. Nat Rev Neurosci 2010;11:589-599.

2. Curcio G, Ferrara M, De Gennaro L. Sleep loss, learning capacity and academic performance. Sleep Med Rev 2006;10:323-337.

3. Cirelli C, Tononi G. Is sleep essential? PLoS Biol 2008;6:e216.

4. Crisp AH. Sleep, activity, nutrition and mood. Br J Psychiatry 1980;137:1-7.

5. Szuba MP, Baxter LR Jr, Fairbanks LA, Guze BH, Schwartz JM. Effects of partial sleep deprivation on the diurnal variation of mood and motor activity in major depression. Biol Psychiatry 1991;30:817-829.

6. Yoo SS, Hu PT, Gujar N, Jolesz FA, Walker MP. A deficit in the ability to form new human memories without sleep. Nat Neurosci 2007;10:385-392.

7. Smith C. Sleep states and learning: a review of the animal literature. Neurosci Biobehav Rev 1985;9:157-168.

8. Guan Z, Peng X, Fang J. Sleep deprivation impairs spatial memory and decreases extracellular signal-regulated kinase phosphorylation in the hippocampus. Brain Res 2004;1018:38-47.

9. Pilcher JJ, Huffcutt AI. Effects of sleep deprivation on performance: a metaanalysis. Sleep 1996;19:318-326.

10. Oswald I. Sleep as restorative process: human clues. Prog Brain Res 1980; 53:279-288.

11. Navara KJ, Nelson RJ. The dark side of light at night: physiological, epidemiological, and ecological consequences. J Pineal Res 2007;43:215-224.

12. Fonken LK, Kitsmiller E, Smale L, Nelson RJ. Dim nighttime light impairs cognition and provokes depressive-like responses in a diurnal rodent. J Biol Rhythms 2012;27:319-327.

13. Cho CH, Lee HJ, Yoon HK, Kang SG, Bok KN, Jung KY, et al. Exposure to dim artificial light at night increases REM sleep and awakenings in humans. Chronobiol Int 2016;33:117-123.

14. Schuhfried G. Vienna test system manual. Mödling: Schuhfried; 1994.

15. Cho AR, Cho CH, Yoon HK, Moon JH, Lee HJ, Kim L. Impact of dim artificial light at night (dALAN) exposure during sleep on human fatigue. Sleep Med Psychophysiol 2016;23:53-60.

16. Philibert I. Sleep loss and performance in residents and nonphysicians: a meta-analytic examination. Sleep 2005;28:1392-1402.

17. Gooley JJ, Chamberlain K, Smith KA, Khalsa SB, Rajaratnam SM, Van Reen E, et al. Exposure to room light before bedtime suppresses melatonin onset 
and shortens melatonin duration in humans. J Clin Endocrinol Metab 2011; 96:E463-E472

18. Kang SG, Yoon HK, Cho CH, Kwon S, Kang J, Park YM, et al. Decrease in fMRI brain activation during working memory performed after sleeping under 10 lux light. Sci Rep 2016;6:36731.

19. Skurvydas A, Zlibinaite L, Solianik R, Brazaitis M, Valanciene D, Baranauskiene $\mathrm{N}$, et al. One night of sleep deprivation impairs executive function but does not affect psychomotor or motor performance. Biol Sport 2020; 37:7-14.

20. Lee HJ, Kim L, Suh KY. The effects of total sleep deprivation on neurocogni- tive functions. J Korean Neuropsychiatr Assoc 1999;38:480-490.

21. Ha TH, You HI, Yoon HY, Song JY, Hong KS, Jeong DU, et al. Validity test and clinical application of computerized standard progressive matrices in Korean patients with mental disorders. J Korean Neuropsychiatr Assoc 1998; 37:1267-1276.

22. Ford CV, Wentz DK. The internship year: a study of sleep, mood states, and psychophysiologic parameters. South Med J 1984;77:1435-1442.

23. Storer JS, Floyd HH, Gill WL, Giusti CW, Ginsberg H. Effects of sleep deprivation on cognitive ability and skills of pediatrics residents. Acad Med 1989; 64:29-32. 